\title{
Polymyalgia rheumatica
}

\section{Assessment of disease activity using erythrocyte sedimentation rate and plasma viscosity}

\author{
W. ESSELINCKX, R. C. BUCKNALL, AND A. ST. J. DIXON \\ From the Royal National Hospital for Rheumatic Diseases, Upper Borough Walls, Bath BAI IRL
}

SUMMARY Comparison of clinically assessed activity of disease with 112 paired readings of the erythrocyte sedimentation rate (ESR) and the plasma viscosity (PV) in 23 patients with polymyalgio rheumatica (PMR) showed the following. (1) A correlation between ESR and PV in both sexescy reaching the significance obtained in a comparison group of patients with rheumatoid arthritis (RA) (109 paired readings), with no significant difference between the PMR and RA groups on analysis of variance of the regression slopes. (2) A degree of scatter of readings around the regression lines so that they could not be used for prediction of ESR from the PV or vice versa. (3) $\chi^{2}$ analysig of normal and abnormal values of ESR and PV which showed a highly significant correlation $\frac{\Phi}{3}$ However 10 readings were abnormally high for ESR in the presence of a normal PV. 5 of these 10 observations were associated with clinical features of disease activity. 20 readings were abnormall $y^{\frac{1}{2}}$ high for PV in spite of a normal ESR with only one instance of clinical activity. These data indic\&te that it is not possible to provide exact guidelines for a 'safe' level of ESR or PV applicable to the individual patient, and measurement of both these indices of disease activity is recommended.

Corticosteroid treatment used to treat active polymyalgia rheumatica (PMR) and to avoid complications such as blindness secondary to associated arteritis (Easterbrook et al., 1967; Wadman and Werner, 1967) may itself induce adverse effects such as compression fractures of vertebrae. Clearly a reliable laboratory test of disease activity is required. The erythrocyte sedimentation rate (ESR) has been widely used but occasional patients with active PMR have a normal ESR and in some of these, active, biopsy-proven arteritis has been reported (Dick and Freeman, 1940; Roux, 1954; Bruk, 1967; A. St. J. Dixon, unpublished; Rynes et al., 1977).

Plasma viscosity (PV) has supplanted ESR in many laboratories as a routine test of disease activity since it can be automated and the results are not influenced by age, sex, or haematocrit (Eastham and Morgan, 1965). Lawrence (1961) and Harkness (1971) have suggested that it might be a more sensitive indicator of disease activity than the ESR. As soon as the PV was proposed as a substitute for the ESR in our laboratory we performed a few comparative measurements on patients with PMR and met some discrepancies between ESR, PV, and

Accepted for publication March 28, 1977

Correspondence to Dr. A. St. J. Dixon clinical activity of disease. This prompted us tळ undertake a more extensive comparison, the result of which are reported here, in parallel with thos $\overrightarrow{8}$ obtained in a group of patients with rheumatoid arthritis (RA).

\section{Patients and methods}

ESR (mm in 1 hour) was measured by the Westergren method. We accepted $20 \mathrm{~mm}$ as the upper limit of normal for males and $30 \mathrm{~mm}$ for females aged 50 o more (Böttiger and Svedberg, 1967). PV was measured using the Coulter viscometer (normal range 1.5-1.72 centipoises). 112 paired readings of ESE and PV were obtained from 23 patients with PMR (46 in males, 66 in females). Disease activity waß also assessed clinically by morning stiffness, pain on movement, and general well being at the time of each venesection. 109 paired readings of ESR ang PV were obtained from 53 patients with RA (40 in males, 69 in females). In both groups all patient were aged 50 years or more and had a haemoglobi concentration of $11 \mathrm{~g} / \mathrm{dl}$ or more. No patient was polycythaemic.

The following statistical analyses were madet (a) Calculation of the correlation coefficient and of 
regression lines in both sexes in both groups and analysis of variance between RA and PMR on the regression lines. (b) Analyses of $X^{2}$ with Yates's correction, except in the males with RA, when a Fisher's exact test was used because of the small number of observations in the extreme positions.

\section{Results}

\section{P M R PATIENTS}

A significant correlation between ESR and PV was found ( $\mathrm{r}=0.59 ; \mathrm{P}<0.001)$ for all patients, and for males $(r=0.617)$ and females $(r=0.626)$ calculated separately. A comparison was made between normal and abnormal values using the $\chi^{2}$ test, assuming a normal PV $<1.72 \mathrm{cp}$ and a normal ESR $<20 \mathrm{~mm} / \mathrm{h}$ and $<30 \mathrm{~mm} / \mathrm{h}$ for males and females respectively. There was a better correlation in the female group $\left(X^{2}=11.95 ; P<0.001\right)$ than in the male group $\left(X^{2}=9.89 ; P<0.01\right)$. For both groups combined correlation was more significant $\left(X^{2}=23 \cdot 22\right.$; $P<0.001)$. It is important to note, however, that in 10 paired readings a raised ESR was associated with a normal PV. 2 of these observations referred to one male and 5 of 8 paired observations in females referred to one patient. 5 (1 male, 4 female) of the total 10 paired readings referred to patients who at the time showed clinically active disease as judged by proximal limb stiffness after rest and aching pain in one or both limb girdles, with or without

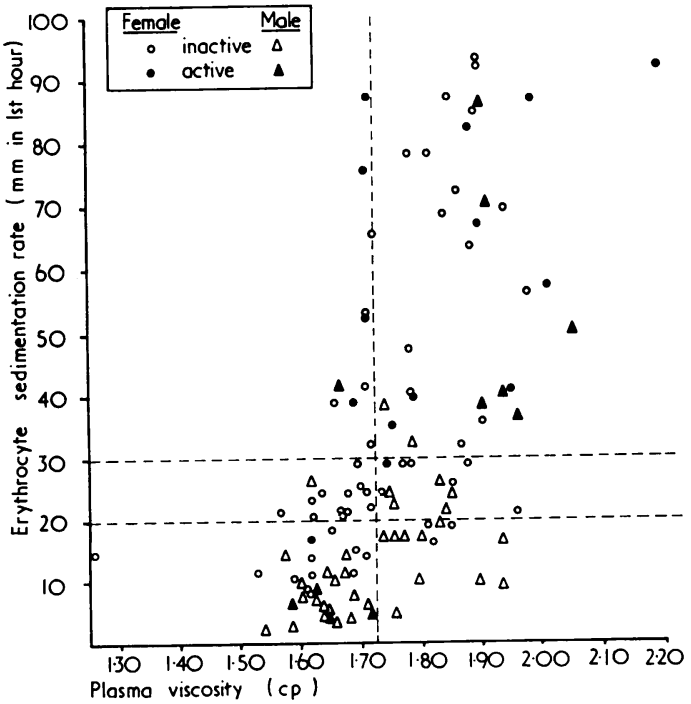

Fig. 112 paired readings of ESR (upper limit of normal. $20 \mathrm{~mm}$ in males and $30 \mathrm{~mm}$ in females) and $P V$ (upper limit of normal 1.72) in 23 patients with $P M R$, all aged 50 years or more. weight loss and anorexia, muscle tenderness, and impaired active movements at shoulders and hips.

In 20 other paired readings (10 in males, 10 in females) a raised PV was associated with a normal ESR. 4 of these paired observations were made in one male patient. Among these patients clinical evidence of disease activity was found in only one.

There were 37 paired readings (13 in males, 24 in females) in which both the ESR and PV were raised. In 23 of these observations (7 in males, 16 in females) there was no clinical evidence of active disease. In 45 paired observations (21 in males, 24 in females) both the PV and ESR were within the normal range. There was clinical evidence of disease activity in 6 of these patients $(4$ male, 2 female).

The regression equations linking ESR with PV were as follows.

\begin{tabular}{llllll}
\hline Male: & PV & $=1.6233$ & +0.0034 & ESR \\
& ESR & $=-162.56+115.13$ & $\times$ PV \\
Female: & PV & $=1.6278$ & +0.0043 & $\times$ ESR \\
& ESR & $=-134.20+87.94$ & $\times$ & PV \\
\hline
\end{tabular}

Examination of the slopes of the prediction lines confirmed that whereas there was no sex difference for PV there was for ESR at all levels. Since scatter around the regression lines was often over a clinically important change in ESR or PV, the equations were not suitable to predict the PV from the ESR and vice versa.

\section{RA PATIENTS}

The correlation between ESR and PV for the whole group was highly significant $(\mathrm{r}=0.678 ; \mathrm{P}<0.001)$ and also when males $(\mathrm{r}=0.661 ; \mathrm{P}<0.001)$ and females $(\mathrm{r}=0.691 ; \mathrm{P}<0.001)$ were calculated separately. Discrepancies, with one exception, all occurred in females, and this was shown by the fact that when comparing normal and abnormal values (Fisher's exact test for males and $\chi^{2}$ for females) the significance of the correlation was greater in males $(P<0.001)$ than in females $\left(X^{2}=4 \cdot 18\right.$; $P<0.5)$. The same comparison made for males and females together yielded a significance of $P<0.001$ $\left(X^{2}=21 \cdot 72\right)$.

The regression equations linking ESR and PV were as follows.

\begin{tabular}{llllll}
\hline Male: & PV & $=1.711$ & +0.0035 & $\times$ & ESR \\
Female $:$ & ESR & $=-180.29+124.47$ & $\times$ & PV \\
& PV & $=1.666$ & +0.0037 & $\times$ & ESR \\
& ESR & $=-186.50+130.44$ & $\times$ & PV
\end{tabular}

Analysis of variance of the regression results showed no significant difference $(P>0 \cdot 1)$ between the PMR and RA slopes for the male or female groups. 


\section{Discussion}

Crockson and Crockson (1974) studied ESR and PV as indices of disease activity in patients with RA and showed a highly significant correlation between the two methods. However we concluded from our results that although we could confirm a statistically significant correlation between results of the PV and ESR in both RA and PMR we could not safely predict the PV from the ESR and vice versa. This was borne out by the wide scatter around the regression lines, and by the number of instances where the PV was raised in the presence of a normal ESR and vice versa.

These discrepancies occurred about twice as often in the PMR group as in the RA group. An accurate assessment of disease activity in patients with RA is perhaps of relatively little importance. However the situation is very different in the case of PMR where inadequate corticosteroid therapy may result in a significant morbidity or even mortality.

The PMR patients presenting with a normal ESR in the presence of clinical features of active disease resembled others reported previously (Russell, 1958; Harrison and Bevan, 1967; Paulley and Hughes, 1960; Mowat and Hazleman, 1974; A. St. J. Dixon, unpublished). Among these 7 patients only one had a raised PV, which suggests that this does not represent a more sensitive indicator of disease activity than the ESR in this situation. However the most important aspect of these observations was that 5 patients with active disease assessed clinically showed a raised ESR in the presence of a normal PV. The use of the PV alone to assess corticosteroid dosage was more likely to result in inadequate treatment.

A recent study of the abrupt and gradual withdrawal of corticosteroids from patients with PMR (Esselinckx et al., 1977) showed that patients with clinically inactive disease had a normal PV and ESR, whereas when one or both of these was raised a relapse on attempting to withdraw corticosteroid therapy was much more likely.

We therefore conclude that in the individual patient with PMR one cannot rely on either the PV or ESR, to assess the activity of disease. It is safer to use both tests. It also seems impossible to provide clear guidelines for a safe level of ESR of PV, applicable to all patients with PMR.

We thank Drs. J. A. Cosh and P. A. Bacon for permission to study their patients; Mrs. J. Crocket5 for performing the ESRs and Mrs. E. Collins fo carrying out statistical analysis of the data.

\section{References}

Böttiger, L. E., and Svedberg, C. A. (1967). Normal erythro cyte sedimentation rate and age. British Medical Journal, 20 85-87.

Bruk, M. I. (1967). Articular and vascular manifestations of polymyalgia rheumatica. Annals of the Rheumatic Diseases 26, 103-113.

Crockson, R. A., and Crockson, A. P. (1974). Relationship of the erythrocyte sedimentation rate to viscosity ang plasma proteins in rheumatoid arthritis. Annals of the Rheumatic Diseases, 33, 53-56.

Dick, C. F., and Freeman, G. (1940). Temporal arteritis. Journal of the American Medical Association, 114, 645-647

Easterbrook, W. M., Baxter, D. W., and Martin, J. A (1967). Temporal arteritis, development during indome thacin therapy. Canadian Medical Association Journal, 97 296-299.

Eastham, R. D., and Morgan, E. G. (1965). Plasma viscosity in clinical laboratory practice. Journal of Medical $\mathrm{Kg} b$ oratory Technology, 22, 70-73.

Esselinckx, W., Doherty, S. M., and Dixon, A. St. J. (1977). Polymyalgia rheumatica. Abrupt and gradual withdrewat of prednisolone treatment. Annals of the Rheumbt? Diseases, 36, 219-224.

Harkness, J. (1971). The viscosity of human blood plasmac its measurement in health and disease. Biorheology, 171-193.

Harrison, M. J. G., and Bevan, A. T. (1967). Early symptoms of temporal arteritis. Lancet 2, 638-640.

Lawrence, J. S. (1961). Assessment of Activity of Disease Lewis, London.

Mowat, A. G., and Hazleman, B. L. (1974). Polymyalgio rheumatica-a clinical study with particular reference to arterial disease. Journal of Rheumatology, 1, 190-202.

Paulley, J. W., and Hughes, J. P. (1960). Giant cell arteritis of the aged. British Medical Journal, 2, 1562-1567.

Roux, J. K. (1954). La syndrome de l'arterite temporale Helvetica Medica Acta, 21, Suppl. 34, 1-82.

Russell, R. W. R. (1958). Giant cell arteritis, a review of 35 cases. Quarterly Journal of Medicine, 28, 471-489.

Rynes, R. I., Mika, P., and Bartholomew, L. E. (1977 Development of giant cell (temporal) arteritis in a patien 'adequately' treated for polymyalgia rheumatica. Anna\& of the Rheumatic Diseases, 36, 88-90.

Wadman, B., and Werner, I. (1967). Therapeutic hazards phenylbutazone and oxyphenbutazone in polymyalgja rheumatica. Lancet, 1, 597-598. 